\title{
Some determinantal inequalities for Hadamard and Fan products of matrices
}

Xiaohui Fu ${ }^{1,2^{*}}$ and Yang Liu $^{2}$

"Correspondence:
fxh6662@sina.com
${ }^{1}$ School of Mathematics and
Statistics, Hainan Normal University,
Haikou, 571158, P.R. China
${ }^{2}$ College of Mathematics and
Statistics, Chongqing University,
Chongqing, 401331, P.R. China

\begin{abstract}
In this note, we generalize some determinantal inequalities which are due to Lynn (Proc. Camb. Philos. 60:425-431, 1964), Chen (Linear Algebra Appl. 368:99-106, 2003) and Ando (Linear Multilinear Algebra 8:291-316, 1980).
\end{abstract}

MSC: 47A63; 47A30

Keywords: determinantal inequalities; $\mathrm{H}$-matrix; $\mathrm{M}$-matrix; Hadamard product; Fan product

\section{Introduction}

Let $C^{m \times n}\left(R^{m \times n}\right)$ be the set of all complex (real) matrices and let $\mathbb{M}_{n}^{+}$be the positive definite Hermitian matrices. Let $Z^{n \times n}=\left\{A=\left(a_{i j}\right) \in R^{n \times n}: a_{i j} \leq 0, i \neq j, i, j \in\{1,2, \ldots, n\}\right\}$. For any $A=\left(a_{i j}\right) \in C^{n \times n}$, its associated matrix is defined by $A^{\prime}=\left(\alpha_{i j}\right)$, where $\alpha_{i i}=\left|a_{i i}\right|, \alpha_{i j}=-\left|a_{i j}\right|$ $(i \neq j)$. For $A=\left(a_{i j}\right), B=\left(b_{i j}\right) \in C^{m \times n}$, the Hadamard product of $A$ and $B$ is $A \circ B=\left(a_{i j} b_{i j}\right) \in$ $C^{m \times n}$ while their Fan product $A * B=\left(c_{i j}\right)$ is defined by $c_{i i}=a_{i i} b_{i i}$ and $c_{i j}=-a_{i j} b_{i j}$ for $i \neq j$.

If $A=\left(a_{i j}\right) \in C^{n \times n}$, then the $k \times k$ leading principal submatrix of $A$ is denoted by $A_{k}$ $(k \in\{1,2, \ldots, n\}) . A_{\alpha}$ denotes the principal submatrix of $A$, with indices in $\alpha \subseteq\{1,2, \ldots, n\}$. $A \in R^{n \times n}$ is called an $M$-matrix if $A \in Z^{n \times n}$ and $\operatorname{det} A_{k}>0(\forall k \in\{1,2, \ldots, n\})$, and we denote it by $A \in M_{n}$. A matrix $A \in C^{n \times n}$ is called an $H$-matrix if $A^{\prime}$ is an $M$-matrix, and we denote it by $A \in H_{n}$.

Lynn [1], Theorem 3.1, proved the following determinantal inequality for $H$-matrices: if $A, B \in H_{n}$, then

$$
\operatorname{det}(A \circ B)^{\prime}+\operatorname{det} A^{\prime} \operatorname{det} B^{\prime} \geq \prod_{i=1}^{n}\left|b_{i i}\right| \operatorname{det} A^{\prime}+\prod_{i=1}^{n}\left|a_{i i}\right| \operatorname{det} B^{\prime},
$$

i.e.

$$
\operatorname{det}(A \circ B)^{\prime} \geq \operatorname{det} A^{\prime} \operatorname{det} B^{\prime}\left(\frac{\prod_{i=1}^{n}\left|a_{i i}\right|}{\operatorname{det} A^{\prime}}+\frac{\prod_{i=1}^{n}\left|b_{i i}\right|}{\operatorname{det} B^{\prime}}-1\right) .
$$

Chen [2], Theorem 2.7, obtained a determinantal inequality for positive definite matrices: if $A=\left(a_{i j}\right), B=\left(b_{i j}\right) \in \mathbb{M}_{n}^{+}$, then

$$
\operatorname{det}(A \circ B) \geq \operatorname{det} A \operatorname{det} B \prod_{k=2}^{n}\left(\frac{a_{k k} \operatorname{det} A_{k-1}}{\operatorname{det} A_{k}}+\frac{b_{k k} \operatorname{det} B_{k-1}}{\operatorname{det} B_{k}}-1\right) .
$$

(c) $2016 \mathrm{Fu}$ and Liu. This article is distributed under the terms of the Creative Commons Attribution 4.0 International License (http://creativecommons.org/licenses/by/4.0/), which permits unrestricted use, distribution, and reproduction in any medium, provided you give appropriate credit to the original author(s) and the source, provide a link to the Creative Commons license, and indicate if changes were made. 
Lin [3] recently proved that a similar result to the block positive definite matrices holds for the block Hadamard product.

Ando [4], Theorem 5.3, has given the following result: if $A=\left(a_{i j}\right), B=\left(b_{i j}\right)$ are $M$ matrices, then

$$
\begin{aligned}
& \operatorname{det}(A * B)+\operatorname{det} A \cdot \operatorname{det} B \\
& \quad \geq\left(\prod_{i=1}^{n} a_{i i}\right) \cdot \operatorname{det} B+\operatorname{det} A \cdot\left(\prod_{i=1}^{n} b_{i i}\right),
\end{aligned}
$$

i.e.

$$
\operatorname{det}(A * B) \geq \operatorname{det} A \operatorname{det} B\left(\frac{\prod_{i=1}^{n} a_{i i}}{\operatorname{det} A}+\frac{\prod_{i=1}^{n} b_{i i}}{\operatorname{det} B}-1\right)
$$

In this paper, we will present some determinantal inequalities for matrices which are generalizations of (1.1), (1.2), and (1.3).

\section{Main results and some remarks}

We give some lemmas before we present the main theorems of this paper.

Lemma 1 ([4], Corollary 4.1.2) Let $A=\left(a_{i j}\right) \in R^{n \times n}$ be an M-matrix. If $\alpha_{i} \subseteq\{1,2, \ldots, n\}$ $(i=1,2,3, \ldots, N)$ satisfies $\alpha_{i} \cap \alpha_{j}=\phi$ for $i \neq j$ and $\bigcup_{j=1} \alpha_{j}=\{1,2, \ldots, n\}$, then

$$
\operatorname{det} A \leq \prod_{i=1}^{N} \operatorname{det}\left(A_{\alpha_{i}}\right) .
$$

In particular,

$$
\operatorname{det} A \leq \prod_{i=1}^{n} a_{i i} .
$$

Lemma 2 ([1], Theorem 3.1) If $A, B$ are $H$-matrices and $C=A \circ B$, then $C$ is $H$-matrix.

Lemma 3 ([5], Theorem 5.2.1) If $A, B$ are positive definite matrices and $C=A \circ B$, then $C$ is positive definite matrix.

Lemma 4 ([6]) If $A, B$ are $M$-matrices and $C=A * B$, then $C$ is $M$-matrix.

Now we present the main results.

First of all, we give a determinantal inequality for the Hadamard product of finite number of $H$-matrices as follows:

Theorem 5 If $A_{1}=\left(a_{1}^{k l}\right), A_{2}=\left(a_{2}^{k l}\right), \ldots, A_{m}=\left(a_{m}^{k l}\right)(k, l=1, \ldots, n)$ are H-matrices, then

$$
\begin{aligned}
\operatorname{det}\left(A_{1} \circ \cdots \circ A_{m}\right)^{\prime} \geq & \operatorname{det}\left(A_{1} \cdots A_{m}\right) \\
& \times\left(\frac{\prod_{i=1}^{n}\left|a_{1}^{i i}\right|}{\operatorname{det} A_{1}^{\prime}}+\cdots+\frac{\prod_{i=1}^{n}\left|a_{m}^{i i}\right|}{\operatorname{det} A_{m}^{\prime}}-(m-1)\right) .
\end{aligned}
$$


Proof By Lemma 2, it is straightforward to observe that the Hadamard product $A_{1} \circ \cdots \circ$ $A_{m}$ is an $H$-matrix. Use induction on $k$. When $k=2$, the result is (1.1). Suppose that (2.2) holds when $k=m-1$

$$
\begin{aligned}
\operatorname{det}\left(A_{1} \circ \cdots \circ A_{m-1}\right)^{\prime} \geq & \operatorname{det}\left(A_{1}^{\prime} \cdots A_{m-1}^{\prime}\right) \\
& \times\left(\frac{\prod_{i=1}^{n}\left|a_{1}^{i i}\right|}{\operatorname{det} A_{1}^{\prime}}+\cdots+\frac{\prod_{i=1}^{n}\left|a_{m-1}^{i i}\right|}{\operatorname{det} A_{m-1}^{\prime}}-(m-2)\right) .
\end{aligned}
$$

When $k=m$, we need to show

$$
\begin{aligned}
\operatorname{det}\left(A_{1} \circ \cdots \circ A_{m}\right)^{\prime} \geq & \operatorname{det}\left(A_{1}^{\prime} \cdots A_{m}^{\prime}\right) \\
& \times\left(\frac{\prod_{i=1}^{n}\left|a_{1}^{i i}\right|}{\operatorname{det} A_{1}^{\prime}}+\cdots+\frac{\prod_{i=1}^{n}\left|a_{m}^{i i}\right|}{\operatorname{det} A_{m}^{\prime}}-(m-1)\right) .
\end{aligned}
$$

By (1.1), we have

$$
\begin{aligned}
\operatorname{det}\left(\left(A_{1} \circ \cdots \circ A_{m-1}\right) \circ A_{m}\right)^{\prime} \geq & \operatorname{det}\left(\left(A_{1}^{\prime} \circ \cdots \circ A_{m-1}^{\prime}\right) A_{m}^{\prime}\right) \\
& \times\left(\frac{\prod_{i=1}^{n}\left(\left|a_{1}^{i i}\right| \cdots\left|a_{m-1}^{i i}\right|\right)}{\operatorname{det}\left(A_{1}^{\prime} \circ \cdots \circ A_{m-1}^{\prime}\right)}+\frac{\prod_{i=1}^{n}\left|a_{m}^{i i}\right|}{\operatorname{det} A_{m}^{\prime}}-1\right) .
\end{aligned}
$$

By the inductive assumption, the above inequality is

$$
\begin{aligned}
\operatorname{det}\left(A_{1} \circ \cdots \circ A_{m}\right)^{\prime} \geq & \operatorname{det}\left(A_{1}^{\prime} \cdots A_{m-1}^{\prime}\right) \operatorname{det} A_{m}^{\prime} \\
& \times\left(\frac{\prod_{i=1}^{n}\left|a_{1}^{i i}\right|}{\operatorname{det} A_{1}^{\prime}}+\cdots+\frac{\prod_{i=1}^{n}\left|a_{m-1}^{i i}\right|}{\operatorname{det} A_{m-1}^{\prime}}-(m-2)\right) \\
& \times\left(\frac{\prod_{i=1}^{n}\left(\left|a_{1}^{i i}\right| \cdots\left|a_{m-1}^{i i}\right|\right)}{\operatorname{det}\left(A_{1}^{\prime} \circ \cdots \circ A_{m-1}^{\prime}\right)}+\frac{\prod_{i=1}^{n}\left|a_{m}^{i i}\right|}{\operatorname{det} A_{m}^{\prime}}-1\right) .
\end{aligned}
$$

Let

$$
\begin{aligned}
& a=\left(\frac{\prod_{i=1}^{n}\left|a_{1}^{i i}\right|}{\operatorname{det} A_{1}^{\prime}}+\cdots+\frac{\prod_{i=1}^{n}\left|a_{m-1}^{i i}\right|}{\operatorname{det} A_{m-1}^{\prime}}-(m-2)\right), \\
& b=\left(\frac{\prod_{i=1}^{n}\left(\left|a_{1}^{i i}\right| \cdots\left|a_{m-1}^{i i}\right|\right)}{\operatorname{det}\left(A_{1}^{\prime} \circ \cdots \circ A_{m-1}^{\prime}\right)}+\frac{\prod_{i=1}^{n}\left|a_{m}^{i i}\right|}{\operatorname{det} A_{m}^{\prime}}-1\right) .
\end{aligned}
$$

By (2.1), we have

$$
\begin{aligned}
& \frac{\prod_{i=1}^{n}\left|a_{j}^{i i}\right|}{\operatorname{det} A_{j}^{\prime}} \geq 1, \quad j=1, \ldots, m, \\
& \frac{\prod_{i=1}^{n}\left(\left|a_{1}^{i i}\right| \cdots\left|a_{m-1}^{i i}\right|\right)}{\operatorname{det}\left(A_{1}^{\prime} \circ \cdots \circ A_{m-1}^{\prime}\right)} \geq 1,
\end{aligned}
$$

and so

$$
a, b \geq 1 \text {. }
$$


Thus by $a b \geq a+b-1$ for $a, b \geq 1$, the above inequality (2.3) is

$$
\begin{aligned}
\operatorname{det}\left(A_{1} \circ \cdots \circ A_{m}\right)^{\prime} \geq & \operatorname{det}\left(A_{1}^{\prime} \cdots A_{m-1}^{\prime}\right) \operatorname{det} A_{m}^{\prime} \times a \times b \\
\geq & \operatorname{det}\left(A_{1}^{\prime} \cdots A_{m}^{\prime}\right) \times(a+b-1) \\
\geq & \operatorname{det}\left(A_{1}^{\prime} \cdots A_{m}^{\prime}\right) \\
& \times\left(\frac{\prod_{i=1}^{n}\left|a_{1}^{i i}\right|}{\operatorname{det} A_{1}^{\prime}}+\cdots+\frac{\prod_{i=1}^{n}\left|a_{m}^{i i}\right|}{\operatorname{det} A_{m}^{\prime}}-(m-1)\right) .
\end{aligned}
$$

This completes the proof.

Remark 6 The above inequality in Theorem 5 is a generalization of the inequality (1.1).

Second, we achieve a determinantal inequality for the Hadamard product of positive definite matrices as follows:

Theorem 7 If $A_{i}(i=1, \ldots, m)(m \geq 2)$ are $n \times n$ positive definite matrices, the Hadamard product of $A_{i}=\left(a_{i}^{l t}\right)$ and $A_{j}=\left(a_{j}^{l t}\right)(i \neq j)$ is denoted by $A_{i} \circ A_{j}$, and $A_{i}^{(k)}$ is the $k \times k(k=$ $1,2, \ldots, n)$ leading principal submatrix of $A_{i}$, then

$$
\begin{aligned}
\operatorname{det}\left(A_{1} \circ \cdots \circ A_{m}\right) \geq & \operatorname{det}\left(A_{1} \cdots A_{m}\right) \\
& \times \prod_{\mu=2}^{n}\left(\frac{a_{1}^{\mu \mu} \operatorname{det} A_{1}^{(\mu-1)}}{\operatorname{det} A_{1}^{(\mu)}}+\cdots+\frac{a_{m}^{\mu \mu} \operatorname{det} A_{m}^{(\mu-1)}}{\operatorname{det} A_{m}^{(\mu)}}-(m-1)\right) .
\end{aligned}
$$

Proof By Lemma 3, it is straightforward to see that the Hadamard product $A_{1} \circ \cdots \circ A_{m}$ is a positive definite matrix. Use induction on $m$. When $k=2$, the result is (1.2). Suppose that (2.4) holds when $k=m-1$. We have

$$
\begin{aligned}
\operatorname{det}\left(A_{1} \circ \cdots \circ A_{m-1}\right) \geq & \operatorname{det}\left(A_{1} \cdots A_{m-1}\right) \\
& \times \prod_{\mu=2}^{n}\left(\frac{a_{1}^{\mu \mu} \operatorname{det} A_{1}^{(\mu-1)}}{\operatorname{det} A_{1}^{(\mu)}}+\cdots+\frac{a_{m-1}^{\mu \mu} \operatorname{det} A_{m-1}^{(\mu-1)}}{\operatorname{det} A_{m-1}^{(\mu)}}-(m-2)\right) .
\end{aligned}
$$

When $k=m$, we need to show

$$
\begin{aligned}
\operatorname{det}\left(A_{1} \circ \cdots \circ A_{m}\right) \geq & \operatorname{det}\left(A_{1} \cdots A_{m}\right) \\
& \times \prod_{\mu=2}^{n}\left(\frac{a_{1}^{\mu \mu} \operatorname{det} A_{1}^{(\mu-1)}}{\operatorname{det} A_{1}^{(\mu)}}+\cdots+\frac{a_{m}^{\mu \mu} \operatorname{det} A_{m}^{(\mu-1)}}{\operatorname{det} A_{m}^{(\mu)}}-(m-1)\right) .
\end{aligned}
$$

By (1.2), we have

$$
\begin{aligned}
& \operatorname{det}\left(\left(A_{1} \circ \cdots \circ A_{m-1}\right) \circ A_{m}\right) \\
& \geq \operatorname{det}\left(\left(A_{1} \circ \cdots \circ A_{m-1}\right) A_{m}\right) \\
& \quad \times \prod_{\mu=2}^{n}\left(\frac{\left(a_{1}^{\mu \mu} \cdots a_{m-1}^{\mu \mu}\right) \operatorname{det}\left(A_{1} \circ \cdots \circ A_{m-1}\right)^{(\mu-1)}}{\operatorname{det}\left(A_{1} \circ \cdots \circ A_{m-1}\right)^{(\mu)}}+\frac{a_{m}^{\mu \mu} \operatorname{det} A_{m}^{(\mu-1)}}{\operatorname{det} A_{m}^{(\mu)}}-1\right) .
\end{aligned}
$$


By the inductive assumption, the above inequality is such that

$$
\begin{aligned}
& \operatorname{det}\left(A_{1} \circ \cdots \circ A_{m-1}\right) \operatorname{det} A_{m} \\
& \quad \times \prod_{\mu=2}^{n}\left(\frac{\left(a_{1}^{\mu \mu} \cdots a_{m-1}^{\mu \mu}\right) \operatorname{det}\left(A_{1} \circ \cdots \circ A_{m-1}\right)^{(\mu-1)}}{\operatorname{det}\left(A_{1} \circ \cdots \circ A_{m-1}\right)^{(\mu)}}+\frac{a_{m}^{\mu \mu} \operatorname{det} A_{m}^{(\mu-1)}}{\operatorname{det} A_{m}^{(\mu)}}-1\right) \\
& \geq \operatorname{det}\left(A_{1} \cdots A_{m-1}\right) \operatorname{det} A_{m} \\
& \quad \times \prod_{\mu=2}^{n}\left(\frac{a_{1}^{\mu \mu} \operatorname{det} A_{1}^{(\mu-1)}}{\operatorname{det} A_{1}^{(\mu)}}+\frac{a_{2}^{\mu \mu} \operatorname{det} A_{2}^{(\mu-1)}}{\operatorname{det} A_{2}^{(\mu)}}+\cdots+\frac{a_{m}^{\mu \mu} \operatorname{det} A_{m-1}^{(\mu-1)}}{\operatorname{det} A_{m-1}^{(\mu)}}-(m-2)\right) \\
& \quad \times \prod_{\mu=2}^{n}\left(\frac{\left(a_{1}^{\mu \mu} \cdots a_{m-1}^{\mu \mu}\right) \operatorname{det}\left(A_{1} \circ \cdots \circ A_{m-1}\right)^{(\mu-1)}}{\operatorname{det}\left(A_{1} \circ \cdots \circ A_{m-1}\right)^{(\mu)}}+\frac{a_{m}^{\mu \mu} \operatorname{det} A_{m}^{(\mu-1)}}{\operatorname{det} A_{m}^{(\mu)}}-1\right) .
\end{aligned}
$$

Let

$$
\begin{aligned}
& a_{\mu}=\frac{a_{1}^{\mu \mu} \operatorname{det} A_{1}^{(\mu-1)}}{\operatorname{det} A_{1}^{(\mu)}}+\frac{a_{2}^{\mu \mu} \operatorname{det} A_{2}^{(\mu-1)}}{\operatorname{det} A_{2}^{(\mu)}}+\cdots+\frac{a_{n}^{\mu \mu} \operatorname{det} A_{m-1}^{(\mu-1)}}{\operatorname{det} A_{m-1}^{(\mu)}}-(m-2), \\
& b_{\mu}=\frac{\left(a_{1}^{\mu \mu} \cdots a_{m-1}^{\mu \mu}\right) \operatorname{det}\left(A_{1} \circ \cdots \circ A_{m-1}\right)^{(\mu-1)}}{\operatorname{det}\left(A_{1} \circ \cdots \circ A_{m-1}\right)^{(\mu)}}+\frac{a_{m}^{\mu \mu} \operatorname{det} A_{m}^{(\mu-1)}}{\operatorname{det} A_{m}^{(\mu)}}-1 .
\end{aligned}
$$

By Fischer's inequality [5], p.506, we have

$$
\begin{aligned}
& \frac{a_{i}^{\mu \mu} \operatorname{det} A_{i}^{(\mu-1)}}{\operatorname{det} A_{i}^{(\mu)}} \geq 1, \quad i=1, \ldots, m, \\
& \frac{\left(a_{1}^{\mu \mu} \cdots a_{m-1}^{\mu \mu}\right) \operatorname{det}\left(A_{1} \circ \cdots \circ A_{m-1}\right)^{(\mu-1)}}{\operatorname{det}\left(A_{1} \circ \cdots \circ A_{m-1}\right)^{(\mu)}}-1 \geq 0,
\end{aligned}
$$

and so

$$
a_{\mu}, b_{\mu} \geq 1
$$

Thus by $a_{\mu} b_{\mu} \geq a_{\mu}+b_{\mu}-1$ for $a_{\mu}, b_{\mu} \geq 1$, the above inequality (2.5) is

$$
\begin{aligned}
& \operatorname{det}\left(A_{1} \circ \cdots \circ A_{m}\right) \\
& \geq \operatorname{det}\left(A_{1} \cdots A_{m-1}\right) \operatorname{det} A_{m} \times \prod_{\mu=2}^{n} a_{\mu} b_{\mu} \\
& \geq \operatorname{det}\left(A_{1} \cdots A_{m}\right) \times \prod_{\mu=2}^{n}\left(a_{\mu}+b_{\mu}-1\right) \\
& \geq \operatorname{det}\left(A_{1} \cdots A_{m}\right) \prod_{\mu=2}^{n}\left(\frac{a_{1}^{\mu \mu} \operatorname{det} A_{1}^{(\mu-1)}}{\operatorname{det} A_{1}^{(\mu)}}+\cdots+\frac{a_{m}^{\mu \mu} \operatorname{det} A_{m}^{(\mu-1)}}{\operatorname{det} A_{m}^{(\mu)}}-(m-1)\right) .
\end{aligned}
$$

This completes the proof.

Remark 8 The inequality in Theorem 7 is a generalization of the inequality (1.2). 
Finally, a result on Fan product of $M$-matrices is obtained in the following theorem.

Theorem 9 If $A_{1}=\left(a_{1}^{k l}\right), A_{2}=\left(a_{2}^{k l}\right), \ldots, A_{m}=\left(a_{m}^{k l}\right)(k, l=1, \ldots, n)$ are M-matrices, then

$$
\begin{aligned}
\operatorname{det}\left(A_{1} * \cdots * A_{m}\right) \geq & \operatorname{det}\left(A_{1} \cdots A_{m}\right) \\
& \times\left(\frac{\prod_{i=1}^{n} a_{1}^{i i}}{\operatorname{det} A_{1}}+\cdots+\frac{\prod_{i=1}^{n} a_{m}^{i i}}{\operatorname{det} A_{m}}-(m-1)\right) .
\end{aligned}
$$

Proof By Lemma 4, it is straightforward to see that the Hadamard product $A_{1} * \cdots * A_{m}$ is an $M$-matrix. Use induction on $k$. When $k=2$, the result is (1.3). Let $k=m-1,(2.6)$ holds:

$$
\begin{aligned}
\operatorname{det}\left(A_{1} \circ \cdots \circ A_{m-1}\right) \geq & \operatorname{det}\left(A_{1} \cdots A_{m-1}\right) \\
& \times\left(\frac{\prod_{i=1}^{n} a_{1}^{i i}}{\operatorname{det} A_{1}}+\cdots+\frac{\prod_{i=1}^{n} a_{m-1}^{i i}}{\operatorname{det} A_{m-1}}-(m-2)\right) .
\end{aligned}
$$

When $k=m$, we need to show

$$
\begin{aligned}
\operatorname{det}\left(A_{1} * \cdots * A_{m}\right) \geq & \operatorname{det}\left(A_{1} \cdots A_{m}\right) \\
& \times\left(\frac{\prod_{i=1}^{n} a_{1}^{i i}}{\operatorname{det} A_{1}}+\cdots+\frac{\prod_{i=1}^{n} a_{m}^{i i}}{\operatorname{det} A_{m}}-(m-1)\right) .
\end{aligned}
$$

By (1.3), we have

$$
\begin{aligned}
\operatorname{det}\left(\left(A_{1} * \cdots * A_{m-1}\right) * A_{m}\right) \geq & \operatorname{det}\left(\left(A_{1} * \cdots * A_{m-1}\right) A_{m}\right) \\
& \times\left(\frac{\prod_{i=1}^{n}\left(a_{1}^{i i} \cdots a_{m-1}^{i i}\right)}{\operatorname{det}\left(A_{1} * \cdots * A_{m-1}\right)}+\frac{\prod_{i=1}^{n} a_{m}^{i i}}{\operatorname{det} A_{m}}-1\right) .
\end{aligned}
$$

By the inductive assumption, the above inequality is

$$
\begin{aligned}
\operatorname{det}\left(A_{1} * \cdots * A_{m}\right) \geq & \operatorname{det}\left(A_{1} \cdots A_{m-1}\right) \operatorname{det} A_{m} \\
& \times\left(\frac{\prod_{i=1}^{n} a_{1}^{i i}}{\operatorname{det} A_{1}}+\cdots+\frac{\prod_{i=1}^{n} a_{m-1}^{i i}}{\operatorname{det} A_{m-1}}-(m-2)\right) \\
& \times\left(\frac{\prod_{i=1}^{n}\left(a_{1}^{i i} \cdots a_{m-1}^{i i}\right)}{\operatorname{det}\left(A_{1} * \cdots * A_{m-1}\right)}+\frac{\prod_{i=1}^{n} a_{m}^{i i}}{\operatorname{det} A_{m}}-1\right) .
\end{aligned}
$$

Let

$$
\begin{aligned}
& a=\left(\frac{\prod_{i=1}^{n} a_{1}^{i i}}{\operatorname{det} A_{1}}+\cdots+\frac{\prod_{i=1}^{n} a_{m-1}^{i i}}{\operatorname{det} A_{m-1}}-(m-2)\right), \\
& b=\left(\frac{\prod_{i=1}^{n}\left(a_{1}^{i i} \cdots a_{m-1}^{i i}\right)}{\operatorname{det}\left(A_{1} \circ \cdots \circ A_{m-1}\right)}+\frac{\prod_{i=1}^{n} a_{m}^{i i}}{\operatorname{det} A_{m}}-1\right) .
\end{aligned}
$$

By (2.1), we have

$$
\begin{aligned}
& \frac{\prod_{i=1}^{n} a_{j}^{i i}}{\operatorname{det} A_{j}} \geq 1, \quad j=1, \ldots, m, \\
& \frac{\prod_{i=1}^{n}\left(a_{1}^{i i} \cdots a_{m-1}^{i i}\right)}{\operatorname{det}\left(A_{1} \circ \cdots \circ A_{m-1}\right)} \geq 1,
\end{aligned}
$$


and so

$$
a, b \geq 1 .
$$

So by $a b \geq a+b-1$ for $a, b \geq 1$, the above inequality (2.7) is

$$
\begin{aligned}
\operatorname{det}\left(A_{1} * \cdots * A_{m}\right) \geq & \operatorname{det}\left(A_{1} \cdots A_{m}\right) \\
& \times\left(\frac{\prod_{i=1}^{n} a_{1}^{i i}}{\operatorname{det} A_{1}}+\cdots+\frac{\prod_{i=1}^{n} a_{m}^{i i}}{\operatorname{det} A_{m}}-(m-1)\right) .
\end{aligned}
$$

This completes the proof.

Remark 10 The inequality in Theorem 9 is a generalization of the inequality (1.3).

Competing interests

The authors declare to have no competing interests.

\section{Authors' contributions}

Xiaohui Fu carried out all the proofs of the results and gave the generalizations of Fan product. Yang Liu participated in the design of the study and drafted the manuscript. All authors read and approved the final manuscript.

\section{Acknowledgements}

We are grateful to Dr. Limin Zou for fruitful discussions. This research was supported by the key project of the applied mathematics of Hainan Normal University, the natural science foundation of Hainan Province (No. 20161005), the Chongqing Graduate Student Research Innovation Project (No. CYS14020) and the Doctoral scientific research foundation of Hainan Normal University.

Received: 22 June 2016 Accepted: 13 October 2016 Published online: 24 October 2016

\section{References}

1. Lynn, MS: On the Schur product of the $\mathrm{H}$-matrices and non-negative matrices and related inequalities. Proc. Camb. Philos. 60, 425-431 (1964)

2. Chen, S: Some determinantal inequalities for Hadamard product of matrices. Linear Algebra Appl. 368, 99-106 (2003)

3. Lin, M: An Oppenheim type inequality for a block Hadamard product. Linear Algebra Appl. 452, 1-6 (2014)

4. Ando, T: Inequalities for M-matrices. Linear Multilinear Algebra 8, 291-316 (1980)

5. Horn, RA, Johnson, CR: Matrix Analysis, 2nd edn. Cambridge University Press, Cambridge (2013)

6. Fan, K: Note on M-matrices. Indag. Math. 67, 43-49 (1964)

\section{Submit your manuscript to a SpringerOpen ${ }^{\odot}$ journal and benefit from:}

- Convenient online submission

Rigorous peer review

- Immediate publication on acceptance

- Open access: articles freely available online

- High visibility within the field

- Retaining the copyright to your article 